How to reference this article

Kłos, A. (2019). Alla ricerca di un capolavoro. II teatro a la traduzione nelle lettere inedite di Sibilla Aleramo a Emilia Szenwicowa. Italica Wratislaviensia, 10(2), 239-256.

DOI: http://dx.doi.org/10.15804/IW.2019.10.1.27

\author{
Anita Kłos \\ Uniwersytet Marii Curie-Skłodowskiej w Lublinie \\ anita.klos@poczta.umcs.lublin.pl \\ ORCID: 0000-0003-4865-0013
}

\title{
ALLA RICERCA DI UN CAPOLAVORO. IL TEATRO E LA TRADUZIONE NELLE LETTERE INEDITE DI SIBILLA ALERAMO A EMILIA SZENWICOWA
}

\author{
IN SEARCH OF A MASTERPIECE. THEATRE \\ AND TRANSLATION IN THE UNPUBLISHED LETTERS \\ OF SIBILLA ALERAMO TO EMILIA SZENWICOWA
}

\begin{abstract}
Sibilla Aleramo (1876-1960) and Emilia Szenwicowa (1889-1972), Polish journalist and translator, met in 1927: the Italian writer hoped that Szenwicowa would translate her novel Amo dunque sono into Polish. A year later, Aleramo spent a few weeks on vacation in Positano, in the villa of the Szenwic family, where she often returned until the outbreak of World War II. In 1931, thanks to Szenwicowa's mediation, Aleramo undertook the translation of Dom kobiet, a theatrical piece by Zofia Nałkowska, into French and literal Italian, using mediatory versions.

A collection of Aleramo's letters has recently been found in the family archive of the Polish journalist's heirs. The letters, published for the first time in the present paper in fragments, testify to the profound friendship that united the two intellectuals, and document, amongst other things, their various collaborative projects concerning the translation of theatre plays from Polish to Italian. The aim of the paper is to analyse the moments of correspondence between Aleramo and Szenwicowa that refer to the theatre.
\end{abstract}

Keywords: Sibilla Aleramo, Zofia Nałkowska, Emilia Szenwicowa, theatre translation, women's writing 
1l'amicizia che aveva legato la scrittrice Sibilla Aleramo ed Emi-
lia Szenwicowa, giornalista e traduttrice polacca, fino allo scoppio della seconda guerra mondiale, e alle conseguenze letterarie della loro conoscenza sono stati dedicati alcuni saggi (Kłos, 2015a, 2016, 2018a e 2018b, pp. 147-182 e 207-219). Szenwicowa (1889-1972), divulgatrice dell'arte italiana antica e moderna, collaboratrice delle rilevanti riviste polacche di profilo socioculturale e letterario (tra cui importanti periodici femminili, "Bluszcz" e "Kobieta Współczesna"), discendente da una ricca famiglia varsaviana di industriali ebrei, conobbe la scrittrice italiana a Roma, nel 1927 (FA-DB, f. 120), ed avrebbe espresso il desiderio di tradurre in polacco Amo dunque sono (AY, lettera del 26 giugno 1928). Nel 1928 Aleramo soggiornò per la prima volta nella villa della famiglia Szenwic a Positano alta, dove ritornò più volte fino al 1939 (Aleramo, 1979, p. 275). Positano, la villa e l'amica straniera sono ritratte nel romanzo aleramiano Il frustino (Eadem, 1932, pp. 151-152 e 163-164) e in alcune poesie del volume Si alla terra (p.e. Positano e Lunare; Aleramo, 1935), ma il risultato artistico più interessante dell'amicizia tra le due intellettuali sembra La casa delle donne, traduzione indiretta (tramite le versioni mediatorie in francese e in italiano) della pièce Dom kobiet di Zofia Nałkowska (1884-1954), grande protagonista del modernismo polacco, autrice di numerosi romanzi e racconti psicologici, eccellente diarista, unica donna accolta nel 1933 nell'Accademia Polacca della Letteratura ${ }^{1}$. L'estesa produzione prosastica dell'autrice è caratterizzata da uno stile preciso e asciutto e da un grande respiro esistenziale. Nałkowska esordì nel 1906 con il romanzo Kobiety (Le donne), in cui sono già presenti gli elementi distintivi di tutte le sue opere successive: un discreto autobiografismo, l'acume dell'indagine psicologica, la sensibilità sociale e femminista e la precisione analitica nella descrizione del mondo (Borkowska, 2010). L'autrice godette in vita di un duraturo successo artistico, stimata come scrittrice, considerata scopritrice di talenti e incarnazione della perfezione letteraria. Dopo il 1945 e la pubblicazione di Medaliony, una raccolta di racconti influenzata dalla sua partecipazione alla Commissione d'in-

1 Cfr. p.es. Kraskowska, 1999 e 2015; Kirchner, 2011; Zawiszewska, 2015. 
dagine sui crimini nazisti ${ }^{2}$, Nałkowska divenne anche una riconosciuta autorità morale.

Dom kobiet, scritto nel 1929, messo in scena e pubblicato in volume nel 1930, fu l'esordio di Nałkowska come autrice drammatica. La traduzione aleramiana del testo, rimasta a lungo inedita, mai messa in scena mentre Aleramo era in vita, è stata recentemente pubblicata dalla casa editrice Pacini (Nałkowska, 2018).

Gli studi precedenti sui contatti della scrittrice italiana con la cultura polacca sono basati principalmente sui documenti dell'archivio privato dell'autrice, custodito presso la Fondazione Gramsci di Roma ${ }^{3}$. A settembre del 2018, il signor Dany Yazbeck, bisnipote di Szenwicowa, mi ha concesso la visione delle lettere inedite di Sibilla Aleramo indirizzate alla giornalista polacca. Sono una quarantina di missive (30 lettere, 9 cartoline postali a Emilia e una lettera a suo marito Feliks Szenwic, noto giurista e avvocato varsaviano), scritte tra il 1928 e il $1935^{4}$. Uno dei fili conduttori della corrispondenza intercorsa tra le amiche, fitta di confidenze riguardanti la vita sentimentale e la situazione economica della scrittrice, è il teatro. I due principali argomenti teatrali attorno a cui ruota lo scambio di missive tra Aleramo e Szenwicowa sono la traduzione della Casa delle donne e il progetto della giornalista polacca di tradurre in collaborazione con l'autrice di Una donna e rappresentare in Italia alcune opere dei drammaturghi polacchi del Ventennio. Le lettere di Sibilla Aleramo in possesso della famiglia Yazbeck permettono di verificare le ipotesi (Kłos, 2015a, pp. 114-115 e 120-123; Eadem, 2018a, pp. 27-30) sulla genesi e messinscena mancata della Casa delle donne nonché stabilire con precisione la cronologia del lavoro traduttivo aleramiano. Svelano anche i gusti teatrali della scrittrice, osservatrice

${ }^{2}$ L'opera fu tradotta anche in italiano: Nałkowska, 1955 e Nałkowska, 2006.

3 Nel Fondo Aleramo si trovano, fra l'altro, oltre settanta missive di Emilia Szenwicowa, tre lettere di Zofia Nałkowska, il contratto per la traduzione e rappresentazione in Italia della Casa delle donne, nonché due versioni della pièce, manoscritta e dattiloscritta (cfr. Zancan, Pipitone, 2006).

${ }^{4}$ Dopo la morte precoce del marito, avvenuta nel 1936, Szenwicowa si trasferì stabilmente in Italia: i contatti tra le due donne sarebbero diventati più immediati. 
attenta della vita teatrale in Italia, e aiutano a capire i meccanismi di scambio interculturale nel contesto italo-polacco.

Come è stato ormai accennato (Mariani, 1987), il teatro fu per Aleramo un territorio artistico particolare, animato da un'impareggiabile forza espressiva. Sembra opportuno ricordare che dopo aver assistito a una rappresentazione di Casa di bambola a Milano, la scrittrice aveva maturato la decisione di lasciare il marito (Aleramo, 1979, p. 14). Invece in una significativa nota di taccuino del 1928 scrisse: "Un'opera di teatro, un'opera di teatro, ecco una delle poche cose, se non forse l'unica, che bramerei ancora fare, ma fare in grande! Sarebbe curioso se tutta la mia vita dovesse concludersi in una tale creazione» (FA-T).

Il sogno del teatro nutrito da un'autrice come lei dotata di un temperamento piuttosto "ateatrale" (Cardarelli, 1969), può sembrare sorprendente. Nonostante le vaste conoscenze nel mondo dello spettacolo, ai casi studiati di Giacinta Pezzana ed Eleonora Duse (Mariani, 1987 e 1988) va aggiunto quello dell'attrice e traduttrice di Una donna in polacco, Soava Gallone, che iniziò a recitare nel teatro per diventare poi una grande diva del cinema muto (Kłos, 2015b), i tentativi di Aleramo di proporsi come un'autrice drammatica per lo più fallirono. Due dei suoi testi teatrali, L'assurdo (1910) e Francesca Diamante. Mistero drammatico in tre atti (1924), rimasero manoscritti, mentre Endimione, messo in scena a Parigi nel 1923, e l'anno seguente a Torino e a Roma per la regia di Tatiana Pavlova, non compreso e mal recitato, fu un clamoroso insuccesso (Mariani, 1987, pp. 123-129). Comunque il ricordo aleramiano della prima parigina di Endimione aiuta a capire quali fossero le ambizioni teatrali della scrittrice:

Di repente, avvertii il silenzio profondo ch'era nella sala, meraviglioso silenzio dove andavano a posarsi impalpabili le parole che scendevano dal palcoscenico. Quali parole? Mie, erano mie, dette da due voci che erano due strumenti, per cento e cento anime dalla vita sospesa ad ascoltare. Mie le parole, mie le anime. (1997, p. 56)

Nel teatro, quindi, l'autore è dotato di uno straordinario, quasi magico potere di imporre la sua voce agli spettatori. Per la donna-scrittrice, alla quale storicamente è stato a lungo negato il diritto di esprimersi, 
il fatto di poter parlare in pubblico ed essere ascoltata ha un'enorme valenza simbolica e trasgressiva (Didier, 1981). Nella pièce di Nałkowska, opera ricca di sfumature autobiografiche (Kirchner, 2011, p. 321), quest'effetto eversivo è moltiplicato. L'opera mette in scena esclusivamente protagoniste femminili, abitanti di una casa di campagna di proprietà della Nonna, matriarca di una famiglia trigenerazionale di sole donne, tutte vecchie (o quasi), tutte vedove o divorziate. Una di loro, Giovanna, è rimasta vedova di recente. Il suo lutto viene interrotto dalla comparsa della giovane Eva, la quale le rivela che suo marito l'aveva tradita sin dai primissimi giorni del matrimonio: dalla sua relazione extraconiugale erano nati tre figli, di cui la stessa Eva è la maggiore. Gettando una nuova luce sulla figura del coniuge defunto, la ragazza costringe Giovanna a confrontarsi con il proprio passato e con il problema ontologico delle "tenebre" che rendono impossibile la conoscenza degli altri (Nałkowska, 2018).

Il successo immediato dell'opera, rappresentata per la prima volta il 21 marzo 1930 al Teatr Polski di Varsavia per la regia di Maria Przybyłko-Potocka (stimata attrice che nella messinscena interpretò il ruolo di Giovanna), fu dovuto in gran parte all'insolito "isolamento femminista", che - come pare - incuriosì anche Sibilla Aleramo, la quale espresse la volontà di tradurre il lavoro in polacco e metterlo in scena in Italia, pur avendo solo un'idea generale della pièce. I contatti tra lei e Nałkowska furono mediati da Szenwicowa, che si impegnò nello sbrigare le formalità necessarie (la prima lettera della giornalista polacca in cui emerge il tema della Casa delle donne è datata 14 novembre 1930; FA-C, lettera 585.345). Szenwicowa non fu scoraggiata neppure dal fatto che l'opera di Nałkowska fosse stata già tradotta in italiano da una certa Maria Poznańska, forse collaboratrice di "Kobieta Współczesna". Nałkowska, che teneva molto alla qualità artistica delle versioni straniere delle sue opere e si lamentava nel diario dell' 'intelligenza insufficiente» e delle scarse capacità artistiche di alcuni suoi traduttori (Nałkowska, 1988 , p. 294), vide nella collaborazione con Aleramo una chance per dare alla Casa delle donne un'adeguata forma letteraria. Ė interessante che il compito assegnato ufficialmente all'autrice di Una donna fosse la revisione linguistica e la "messa a punto" letteraria della traduzione 
di Poznańska, artisticamente imperfetta e piena di inesattezze lessicali (FA-C, lettera 585.354).

Le lettere di Aleramo all'amica polacca gettano una nuova luce sugli aspetti organizzativi e legali dell'operazione traduttoria. Sembra indicativa la precauzione della scrittrice di non prendere obblighi, neanche informali, senza aver letto il copione francese della pièce. Nella missiva aleramiana del 27 novembre 1930 (AY) leggiamo:

Non ho ancora ricevuto nulla dalla Sig. Posnanska [sic!] né dalla Sig. Navkowska [sic!], e perciò non ho ancora risposto a Picasso. Mi pare che la cosa più urgente e più semplice sarebbe quella di mandarmi la traduzione francese (se è stampata) così tanto io che Picasso potremo subito decidere se il lavoro va o no per l'Italia. Cerca dunque cara, di farmi avere al più presto questa traduzione francese, mentre prosegui le trattative con l'autrice e la traduttrice.

La traduzione francese di Dom kobiet, La maison des femmes, era stata eseguita nel 1930 da Thérèse Koerner, apprezzata traduttrice dal polacco al francese, per la progettata rappresentazione parigina che, a quanto risulta, non ebbe mai luogo (Kłos 2018b, pp. 215-217). Nałkowska, che reputava Koerner «un'artista eccellente», ma allo stesso tempo la considerava una pessima collaboratrice, attribuendole addirittura la responsabilità personale di aver rovinato le «congiunture» parigine per la pièce (Nałkowska, 1988, pp. 171-172), inviò a Roma il dattiloscritto di La maison des femmes come eventuale aiuto nel lavoro di revisione (FA-C, lettera 585.354). Dopo la lettura dell'opera, eccellente secondo la sua opinione, Aleramo iniziò le difficili trattative per aumentare la cifra degli attesi profitti e riuscì a ottenere dall'autrice polacca la garanzia del $30 \%$ del guadagno ricavato dalla messinscena italiana:

Ho ricevuto il copione francese della Casa delle Donne, e una lettera amabile dell'autrice, e un'altra della traduttrice.

Il lavoro è e c cellente, mi piace moltissimo, e lo scriverò oggi stesso all'autrice.

Però, cara, l'autorizzazione che m'ha mandata la sig.ra Nałkowska non è sufficiente per tutelarmi. E prima d'iniziare le trattative, sia con Picasso, 
che con altri, occorre ottenere dalla sig.ra Nalkowska [sic!] una c on c e ssi o ne più ampia e chiara.

Milecska [sic!] mia, vuoi aver tanta bontà da intercedere ancora presso la sig.ra Nalkowska [sic!] e presso la sig.na Posnanska [sic!] per ottenere che firmino una delle due copie del contratto che qui ti mando, e accettino l'altra firmata da me, come garanzia?

Se credi, e s'egli ha un minuto di tempo, fa leggere al signor Felice, che è avvocato, questo contratto, perché ti dica che gli pare che vada bene.

Cerca di far capire alla sig.na Posnanska [sic!], che il mio lavoro, di r e visione e di mise au point della sua traduzione, e quello di trattare e per combinare qui per la rappresentazione, e infine il mio nome conosciuto, col quale firmerei con lei la traduzione, rendono questa mia richiesta del $30 \%$ anziché del $25 \%$ (ossia $30 \%$ a me e $20 \%$ a 1 ei). [...] Soltanto quando avrò questo contratto firmato, potrò far leggere a Picasso e ad altri il copione francese: prima no!

Appena poi avrò concluso con Picasso od altro direttore, mi metterò al lavoro di revisione della traduzione (che ancora non ho ricevuta).

Aleramo sin dall'inizio pensò a coinvolgere per la messinscena Lamberto Picasso (1880-1962), noto attore e capocomico, collaboratore di Luigi Pirandello e "direttore sostituto" della pirandelliana Compagnia del Teatro d'Arte, incontrato nel 1925 in occasione della rappresentazione della sua versione del Pèlerin di Charles Vildrac durante l'ultimo dei "giovedì del Teatro d'Arte" all'Odeschalchi di Roma: Picasso vi aveva interpretato la parte del protagonista, Edoardo (Aveline, Cassou, 1983, pp. 129-131). L'attore apparteneva ai «convinti assertori della necessità di un teatro d'arte in Italia», e non trovando nelle compagnie esistenti un posto corrispondente alle sue ambizioni artistiche, mise in atto alcune iniziative in proprio (Tinterri, 2009, p. 13, n. 1). Nel 1930, con la sua troupe, portò in scena al Teatro Valle di Roma Il grande viaggio del drammaturgo inglese Robert Cedric Sherriff (1896-1975). La rappresentazione romana della pièce, con soli protagonisti maschili, costituì un grande successo di Picasso, che ne fu regista e attore (Ibidem, pp. 113-119). Per approfittare di quel successo il capocomico intraprese una tournée che lo trattenne fuori Roma per vari periodi, il che complicava i progetti delle amiche riguardanti La casa delle donne. Soltanto nella lettera del 19 gennaio 1931 Aleramo comunica a Szenwicowa: «Domani farò leggere a Picasso, che sarà di ritorno, la trad. francese 
del lavoro della Nalkowska (sic!) - e sentiremo la sua opinione, e poi informerò subito la signora, e la traduttrice, e anche te». A causa degli impegni assunti in precedenza Picasso rinunciò a mettere in scena la pièce polacca. Nella missiva all'amica del 26 febbraio 1931 (AY) Aleramo scrive:

Mia Mileczka,

la tua Sibilla ha passato un periodo terribile, è perciò non ti ha più scritto. [...] Per il lavoro di Nalkowska [sic!] mi sono fatta forza e l'ho tradotto ossia messo in buon italiano - l'ho fatto leggere a Picasso, che l'ha trovato interessante, ma non può prenderlo ora dovendo continuare la sua tournêe col lavoro inglese sulla guerra. Allora anche per consiglio di gente pratica, l'ho affidato ad un agente, all'Ufficio Editoriale Repertorio Drammatico - che vi si è interessato e spera poter presto combinare con qualche compagnia. Bisognerà però dargli il 10\% sugli utili, pazienza. Ma tutto questo andrà comunque per le lunghe, ci vorranno dei mesi, forse sino ad autunno, ed io da qui ad allora sarò morta almeno dieci o dodici volte!...

La complessa operazione interpretativa, nella quale Aleramo accosta brani della versione di Poznańska (laddove necessario corretti o modificati stilisticamente) ai brani della traduzione francese di Koerner riscritti, di volta in volta, in italiano, è stata analizzata specificamente in altre occasioni ${ }^{5}$. Non è invece chiaro chi fossero la "gente pratica» e l'«agente» a cui la scrittrice aveva affidato il compito di trovare un esecutore de La casa delle donne. Il fatto è che pochi mesi dopo la dichiarata assunzione dell'agente istituzionale Aleramo ritornò a esplorare canali privati nel mondo dello spettacolo, tentando di convincere Tatiana Pavlova (1894-1975), grande attrice e regista di origini russe, che nel 1924 aveva messo in scena l'Endimione (Aleramo, 1997, pp. 218-220), a rappresentare anche l'opera dell'autrice polacca. In un laconico messaggio datato 23 luglio 1931, Pavlova parla indubbiamente de La casa delle donne: "so del lavoro da tempo dai giornali francesi e del successo pero [sic!] non eccessivo a Varsavia, ma il lavoro m'interessa» (FA-C,

5 P.es. in Kłos, 2018a, pp. 19-27, e 2018b, pp. 219-246. 
lettera 597.191). Eppure, per ragioni sconosciute, la collaborazione con la regista non si realizzò.

Secondo il contratto stipulato con l'autrice, Aleramo era stata autorizzata a far rappresentare La casa delle donne in Italia entro la fine del 1932. I tentativi di portare sui palcoscenici italiani la pièce di Nałkowska coincisero con trasformazioni importanti dell'organizzazione teatrale nell'Italia fascista, come istituzione della Corporazione dello Spettacolo (1930) e la nuova legge sulla censura del 1931, che attribuiva competenze censoriali all'amministrazione centrale dello Stato ${ }^{6}$. Nell'inventario dei copioni teatrali per cui è stata richiesta l'autorizzazione dell'Ufficio censura teatrale (Ferrara, 2004), non troviamo La casa delle donne, tantomeno tra i titoli proposti dalle compagnie di Picasso e Pavlova ${ }^{7}$, per lo più commedie. In una lettera del 1934 l'instancabile Szenwicowa, citando notizia sulla prima di una pièce di Nałkowska a Parigi, suggerì all'amica di riproporre il copione de La casa delle donne a Pavlova oppure di mandarlo a un noto attore e capocomico, Ruggero Ruggeri (1871-1953). Non sappiamo se la scrittrice effettivamente lo fece - fatto sta che La casa delle donne per molti anni cadde in oblio. Ma grazie alla domanda dell'amica polacca che riguardava Ruggeri, si viene a sapere che Aleramo partecipò al famoso Convegno Volta sul teatro drammatico di Roma, un'occasione di propaganda internazionale per il regime fascista (Fried, 2014), e vide l'altrettanto celebre rappresentazione della Figlia di Iorio di Gabriele D'Annunzio, proposta come una testimonianza della rinascita della regia teatrale in Italia (Pieri, 2017). Nella messinscena, erano coinvolti i grandi della cultura teatrale dell'epoca - Luigi Pirandello come regista, Marta Abba e Ruggeri come coppia di protagonisti, insieme al più apprezzato pittore italiano allora vivente, Giorgio de Chirico, cooptato come scenografo. Eppure i linguaggi artistici di tutte quelle individualità rimasero nello spettacolo «reciprocamente irrelati»,

${ }^{6}$ E in effetti ad una sola persona, il prefetto Leopoldo Zurlo; si vedano fra l'altro Scarpellini, 1989; Thompson, 1996, Ferrara, 2004.

7 L'unico lavoro polacco nell'inventario, che abbraccia gli anni 1931-1944, è Dramma di Monica (pol. Sprawa Moniki, 1931), pièce femminista di Maria MorozowiczSzczepkowska (1885-1968) nella riduzione di Maria Bazzi (1897-1959), respinto dalla censura fascista (Ferrara, 2004, vol. 1, p. 60; vol. 2, p. 610). 
dando come risultato «teatro non buono», come avrebbe detto diplomaticamente il grande Gordon Craig (Ibidem, p. 35). Aleramo parla apertamente di un «disastro»:

Qui son stata per una settimana assai occupata col Congresso del Teatro. Stefan Zweig non c'era. Ma c'era Maeterlinck, con la moglie, e ambedue s'erano innamorati di me. Lo vedrai dalle belle dediche che mi hanno lasciate.

Non ho potuto parlare con Ruggeri! La rappresentazione della Figlia di Iorio è stata un disastro. Ti racconterò a voce. (AY, lettera del 19 ottobre 1934)

Contemporaneamente alle relazioni sulle vicende de La casa delle donne, nella corrispondenza delle amiche si sviluppa il tema delle rappresentazioni italiane di diversi lavori per teatro polacchi. Sperando nel successo dell'opera di Nałkowska sui palcoscenici in Italia Szenwicowa propose all'amica italiana una specie di costante collaborazione traduttoria. Lei avrebbe eseguito la prima versione delle traduzioni di pièces dei drammaturghi polacchi contemporanei e ad Aleramo sarebbe spettata la correzione letteraria di esse (Kłos, 2016, p. 113). La cooperazione traduttiva doveva inoltre assicurare qualche reddito alla scrittrice italiana che attraversava un periodo di notevoli difficoltà economiche. La sua situazione finanziaria, sempre precaria dopo la separazione dal marito, si era radicalmente aggravata dopo lo scandalo suscitato dall'articolo Ritorno Ondina pubblicato a sul "Corriere della Sera" nel settembre del 1928: di conseguenza il giornale aveva sospeso la collaborazione ${ }^{8}$

Nella lettera del 6 marzo 1931 (AY) Sibilla espone non poche obiezioni all'idea dell'amica:

Circa le traduzioni che affettuosamente ti proponi di fare con me, ti prevengo che è difficilissimo collocare delle opere teatrali (il teatro in Italia è in una situazione tragica) a meno che non siano c a p o la v o ri . Forse riuscirò a far dare La Casa delle Donne, per la curiosità d'un lavoro ove i personaggi son

8 «Ricevetti al mattino una lettera del Corriere, penosissima (Ricordi i miei presentimenti, a Positano?) A quanto pare, l'articolo Ondina ha fatto scandalo e mi pregano di sospendere la collaborazione!!» (AY, lettera del 15 settembre 1928). 
tutti donne. Ma ci vorranno, prima, mesi e forse s e me stri !! Intanto io potrò appiccarmi dieci volte a queste travi.

Nelle lettere di Aleramo ritorna quasi ritualmente il tema della crisi dell'industria teatrale, punto fermo anche del dibattito critico sul teatro in Italia dal primo dopoguerra in poi. A fronte della continua ascesa del cinema, verso la fine degli anni Venti si faceva sempre più evidente la recessione del settore, con il calo di incassi, una generale mediocrità artistica degli allestimenti e diverse fragilità organizzative del sistema ${ }^{9}$ nonché la necessità di una riforma. Proprio a gennaio del 1931 Silvio d'Amico aveva pubblicato su "Pegaso" il famoso saggio La crisi del teatro (Orecchia, 2012, pp. 285-311). Comunque, nonostante la poca fede nel successo delle traduzioni sul "difficilissimo" mercato italiano, Sibilla decise di aiutare Emilia nella ricerca di un capolavoro del teatro polacco che potesse essere collocato sulle scene italiane. Tra i drammaturghi potenzialmente interessanti per il pubblico straniero Szenwicowa nomina Tadeusz Rittner e Gabriela Zapolska (FA-C, lettera 602.336), eppure finì a mandare all'amica i copioni francesi di due autori ben diversi: Pod falami (Sotto le onde; 1931) di Jan Adolf Hertz (1878-1943), artista oggi completamente dimenticato, ma all'epoca direttore del Sindacato polacco degli autori drammatici, e Adwokat i róże (L'avvocato e le Rose) di Jerzy Szaniawski (1886-1970), nel corso del Novecento diventato classico della drammaturgia polacca ${ }^{10}$. Rittner (1873-1921), scrittore bilingue polacco-tedesco abitante a Vienna, combinò la carriera letteraria con quella di un alto funzionario di Stato asburgico (Milanowski, 1999; Kubiszyn-Mędrala, 2016). Le sue opere per il teatro (tra cui $W$ matym domku (In una casa piccola), parodiato da Stanisław Ignacy Witkiewicz nella sua famosa pièce In una casa di campagna), con un profondo interesse verso la psicologia dei personaggi, si accostano alla poetica naturalista. Convenzionalmente classificate come commedie, finiscono di solito con la sconfitta del protagonista. Anche la scrittura di Zapolska (1857-1921), con forti elementi di critica sociale, nasce

9 Cfr. p.es. Scarpellini, 1989, pp. 116-128.

10 La sua visione del teatro viene spesso accostata dai comparatisiti polacchi a quella di Pirandello, cfr. p.es. Bronowski, 2008, pp. 80-85; Kucharuk, 2011. 
dal naturalismo. Attrice e scandalista, pubblicista e autrice prolifica di romanzi e opere drammatiche, nella cultura polacca è diventata sinonimo di lotta contro ipocrisia e doppia morale della società borghese ${ }^{11}$. Sembra interessante menzionare che la pièce più famosa dell'autrice polacca, La morale della Signora Dulska, fu messa in scena nel 1954 al Teatro Pirandello di Roma per la regia di Lamberto Picasso (Schenone, 1998). Szaniawski, più giovane di Rittner e Zapolska, propone invece un teatro filosofico, che attinge alle esperienze del simbolismo modernista europeo. Nelle sue opere la realtà si mescola spesso al sogno, creando un'atmosfera poetica e una tensione metafisica.

Hertz, a cui Szenwicowa si era rivolta con una domanda di aiuto, approfittò della sua funzione istituzionale per proporle il suo testo da tradurre (FA-C, lettera 602.380), mentre l'interesse della giornalista per il testo di Szaniawski era profondo e genuino. L'opera, per la prima volta rappresentata nel 1929 al Teatr Nowy di Varsavia per la regia di Aleksander Zelwerowicz, racconta la storia di un avvocato che si ritira dalla professione e si dedica a coltivare le rose. Dopo un susseguirsi di eventi imprevisti, l'uomo è costretto alla revisione delle proprie convinzioni etiche e delle sue scelte esistenziali. La giornalista teneva molto alla traduzione di quella pièce («mi piacerebbe tanto tanto di tradurre L'avvocato e le rose. (...) E una cosa che sento così bene questa idea di nascondersi lontano di tutti fra le rose. E quest'uomo che non vuole rompere l'armonia intorno di se anche se soffre», FA-C, lettera 602.380).

L'autorizzazione per la traduzione e la messinscena italiana di Adwokat i róże fu concessa da Szaniawski a Szenwicowa nel febbraio del 1932, non senza esitazioni e complicazioni. L'autore in primis non aveva avuto «più fiducia di lavorare colle Signore perché in Germania aveva cattive esperienze»; inoltre aveva ormai promesso l'autorizzazione per l'Italia a Leopold Kociemski, traduttore, scrittore e pubblicista, collaboratore dell'Istituto per l'Europa orientale (Santoro, 2005, pp. 153-154) e al traduttore Bolesław Miciński (FA-C, lettere 602.365, $602.380,607.64)$. Szenwicowa riuscì comunque a convincere Szaniawski a cambiare gli accordi iniziali e di cedere a lei i diritti per la

${ }^{11}$ Cfr. p.es. Gawin, 2011. 
rappresentazione italiana: «Sig. Szaniawski mi ha dato l'autorisazione (sic!) perché io (...) ho piaciuto a lui! (...) Ma lui è un vero amore! Non guarda la persona colla quale parla, ma se alza gli occhi si vede un fuoco dell'anima che fa bene al cuore» (FA-C, lettera 607.64).

Dopo aver letto Sulle onde Aleramo subito escluse la possibilità di tradurre in italiano l'opera di Hertz:

Oggi ho letto il lavoro del signor Hertz, Sulle Onde. Devo dirti subito che esso purtroppo n on mi pare tanto interessante da tradurlo per le scene italiane. È un lavoro fine, delicato, ma senza una vera originalità, e sono quasi certa che nessun capocomico vorrà accettarlo, specie in questi tempi difficili, che si va in caccia soltanto di grandi successi.

Basta. Io terrò qui il copione francese, e all'occasione lo farò leggere a qualcuno per averne il parere, poi ti riferirò.

Ora speriamo nell'Avvocato e le Rose. Aspetto con desiderio la traduzione francese, e appena l'avrò letta la spedirò o a Picasso o a Tatiana. Intanto tu incomincia a tradurlo, e appena hai pronto un atto spediscimelo, così mi ci metterò subito anch'io, aiutandomi anche con il copione francese. Per i diritti sta bene quel che hai concluso con l'Autore. Bisognerà mi mandi una copia del contratto per me, firmata dall'autore e da te; e, se vuoi, un'altra copia che ti rimanderò con la mia firma, per la regolarità.

Ma sei poi sicura che il signor Kocinsky [sic!] qui a Roma non sollevi delle difficoltà, se ha già tradotto un atto? (AY, lettera del 3 marzo 1932)

Sin dall'inizio Szenwicowa sosteneva che la pièce era un materiale ideale per Lamberto Picasso e/o Tatiana Pavlova (Kłos 2016, p. 114). Nelle lettere alla scrittrice la giornalista esorta Aleramo in continuazione a stipulare un contratto preliminare per la rappresentazione del lavoro in Italia, programmando anche la possibilità di pubblicare la traduzione della pièce in volume. Eppure, dopo la lettura della versione francese del lavoro di Szaniawski, Aleramo non condivideva l'entusiasmo di Emilia per la pièce, "tutto sfumature", nella quale non vedeva un personaggio femminile forte e visibile:

Cara,

vedi, ti ubbidisco, e ti scrivo subito ciò che penso dell'Avvocato e le Rose, finito di leggere or ora. 
Certo è un opera di profonda finezza e nobiltà spirituale ed artistica. Ma (o è colpa della traduzione francese?) risulta, se si pensa al pubblico specialmente dei teatri italiani, un po' troppo misteriosa, evasiva, troppo poco comprensibile. Nulla è detto, tutto dev'essere indovinato. Non soltanto per ciò che riguarda il dramma centrale, del giovanotto sorpreso nel giardino, e arrestato, ma anche per l'altro giovine amante e per lo stesso avvocato. In quanto alla donna, povera donna, si vede che l'autore ha voluto assolutamente farla inesistente, un ombra qualunque, di cui tutti però si innamorano e per la quale tutti soffrono. La figura della madre è un po' più accennata. Ma, insomma, ho gran paura che il pubblico italiano (e forse anche la critica) faccia il viso dell'arme a un lavoro così sottile, tutto sfumature. Perciò, ripeto, può darsi che rileggendolo nella tua traduzione, io modifichi la mia impressione. Me lo auguro vivamente. E mi metterò d'impegno a correggere il tuo italiano dove ne avrà bisogno. Ti aspetto, cara; ma non tardare, perché ho quasi deciso di andare a Viareggio verso la metà della prossima settimana, per il Premio e per la Fiera. (AY, lettera non datata [luglio 1932])

Sebbene Szenwicowa argomentasse che la donna nella pièce di Szaniawski non era "inesistente" (FA-C, cartolina postale 612.245), non si trovano tracce di archivio che confermino che Aleramo intraprese il lavoro di revisione alla traduzione dell'amica, compiuta, a quanto pare, ad agosto del 1932. Nei mesi successivi Szenwicowa chiese un parere sul testo allo scrittore Corrado Alvaro, conosciuto a Positano, e al noto critico teatrale Eugenio Giovannetti (FA-C, lettera lettera 616.396), ma sembra che per Aleramo L'Avvocato e le rose fosse ormai argomento chiuso.

Le lettere di Aleramo a Szenwicowa testimoniano le aspirazioni teatrali di ambedue le intellettuali che avevano l'ambizione di elaborare direttamente il materiale drammaturgico e di decidere sui contenuti presentati sulle scene. La scrittrice italiana e la giornalista polacca assumono qui anche un importante ruolo come mediatrici interculturali, che scelgono dalle culture straniere quegli elementi e quei valori artistici e sociali che non necessariamente coincidono con i canoni e con le scelte maschili. Le lettere delle due amiche analizzate nel presente saggio confermano anche l'importanza degli studi sugli archivi delle donne che offrono sempre parecchie possibilità di ricerca e rimangono un terreno dove sono ancora possibili scoperte rilevanti. 


\section{ELENCO DELLE ABBREVIAZIONI:}

AY - Archivio privato della famiglia Yazbeck.

FA-C - Fondazione Gramsci onlus, Fondo Aleramo, serie 2: "Corrispondenza" - "Sezione cronologica".

FA-DB - Fondazione Gramsci onlus, Fondo Aleramo, serie 3: "Scritti", sottoserie 7: "Altri scritti", UA 111: "Dati biografici di Sibilla Aleramo per gli esecutori testamentari".

FA-T - Fondazione Gramsci onlus, Fondo Aleramo, serie 3: "Scritti", sottoserie 6: "Note del taccuino", UA 90: "Appunti e note 1928", docc. 7.

\section{BIBLIOGRAFIA}

Aleramo, S. (1932). Il frustino. Romanzo. Milano: Mondadori.

Aleramo, S. (1935). Si alla terra. Nuove poesie (1928-1934). Milano: Mondadori.

Aleramo, S. (1979). Un amore insolito. Diario 1940-1944, a cura di A. Morino. Milano: Feltrinelli.

Aleramo, S. (1997). Andando e stando, a cura di R. Guerricchio. Milano: Feltrinelli.

Aveline, C., \& Cassou J. (1983). Charles Vildrac. Roma, Paris: Bulzoni $\&$ Nizet.

Borkowska, G. (2010). Imperatyw miłości. In Z. Nałkowska, Kobiety (pp. 5-11). Warszawa: Prószyński i S-ka.

Bronowski, C. (2008). Historia dramaturgii włoskiej XX wieku. Wrocław: Wydawnictwo Uniwersytetu Wrocławskiego.

Cardarelli, V. (1969). Endimione di Sibilla Aleramo al Valle (Il Tevere, 24 febbraio 1925). In V. Cardarelli, La poltrona vuota (pp. 218-219). Milano: Rizzoli.

Didier, B. (1981). L'Écriture-femme. Paris: Presses Universitaires de France.

Ferrara, P. (Ed.) (2004). Censura teatrale e fascismo (1931-1944) La storia, l'archivio, l'inventario. Roma: Ministero per i Beni e le Attività Culturali \& Direzione Generale per gli Archivi.

Fried, I. (2014). Il Convegno Volta sul teatro drammatico. Roma 1934. Un evento culturale nell'età dei totalitarismi. Corazzano: Titivillus. 
Gawin, M. (2011). Przeciw emancypacji kobiet. Teksty drugie, 4, 235-252. Kirchner, H. (2011). Natkowska albo życie pisane. Warszawa: W.A.B.

Kłos, A. (2015a). "La casa delle donne". Sulla storia della traduzione italiana di "Dom kobiet" di Zofia Nałkowska. pl.it / rassegna italiana di argomenti polacchi, 6, 107-123.

Kłos, A. (2015b). Tradurre il femminismo. Sulla traduzione polacca di Una donna di Sibilla Aleramo. Kwartalnik Neofilologiczny, LXII/2, 257-265.

Kłos, A. (2016). Ineczka, Mileczka, Positano. Sibilla Aleramo e i suoi contatti con la cultura della Polonia tra le due guerre. Bollettino di italianistica, 1, 93-116.

Kłos, A. (2018a). Introduzione. «Riconoscenza e solidarietà». Sibilla Aleramo e La casa delle donne di Zofia Nałkowska. In Z. Nałkowska, La casa delle donne, trad. di S. Aleramo, con introduzione e a cura di A. Kłos (pp. 5-32). Pisa: Pacini.

Kłos, A. (2018b). Apologia kobiecego ducha. Sibilla Aleramo i jej zwiazki $z$ polska kultura literacka pierwszej połowy XX wieku. Lublin: Wydawnictwo UMCS.

Kraskowska, E. (1999). Zofia Nałkowska. Poznań: Rebis.

Kraskowska, E. (2015). Sama wśród mężczyzn. Zofia Nałkowska jako instytucja życia literackiego. In E. Kraskowska \& B. Kaniewska (Eds.), Polskie pisarstwo kobiet w wieku XX: procesy i gatunki, sytuacje i tematy (pp. 17-28). Poznań: Wydawnictwo UAM.

Kubiszyn-Mędrala, Z. (2016). Z bilingwizmu pisarzy młodopolskich. Antroponimia w polsko- i niemieckojęzycznych dramatach Tadeusza Rittnera. LingVaria, 2, 185-200.

Kucharuk, S. (2011). Pirandello i Szaniawski: przyczynek do badań komparatystycznych. Lublin: Wydawnictwo UMCS.

Mariani, L. (1987). Sibilla Aleramo. Significato di tre incontri col teatro: il personaggio di Nora, Giacinta Pezzana, Eleonora Duse. Teatro e Storia, 2, 67-133.

Mariani, L. (1988). Eleonora Duse e Sibilla Aleramo: un teatro per "la donna nuova”. In A. Buttafuoco \& M. Zancan (Eds.), Svelamento. Sibilla Aleramo: una biografia intellettuale (pp. 208-225). Milano: Feltrinelli.

Milanowski, A. (1999). Czy Tadeusz Rittner był pisarzem polskim czy austriackim? In G. Ritz \& G. Matuszek (Eds.), Recepcja literacka i proces literacki. O polsko-niemieckich kontaktach literackich od modernizmu po okres międzywojenny (pp. 63-85). Kraków: Universitas. 
Nałkowska, Z. (1955). I ragazzi di Oswiecim. Roma: Edizioni di cultura sociale.

Nałkowska, Z. (1988). Dzienniki IV: 1930-1939, vol.1. Warszawa: Czytelnik.

Nałkowska, Z. (2006). Senza dimenticare nulla, trad. di B. Meriggi, a cura di G. De Biase. Napoli: L'Ancora del Mediterraneo.

Nałkowska, Z. (2018). La casa delle donne, trad. di S. Aleramo, con introduzione e a cura di A. Kłos. Pisa: Pacini.

Orecchia, D. (2012). Il critico e l'attore. Silvio D'Amico e la scena italiana di inizio del Novecento. Torino: Accademia University Press.

Pieri, M. (2017). 1934. Il giuoco delle parti dietro le quinte de La Figlia di Iorio. Archivio d'Annunzio, 4, 25-28.

Santoro, S. (2005). L'Italia e l'Europa orientale: diplomazia culturale e propaganda 1918-1943. Milano: FrancoAngeli.

Scarpellini, E. (1989). L'organizzazione teatrale e politica del teatro fascista. Firenze: La Nuova Italia.

Schenone, F. (1998). La rivista "Teatro d'oggi". In A. Tinterri \& N. Pasero (Eds.), La piazza del popolo (pp. 125-163). Roma: Meltemi.

Thompson, D. (1996). The organisation, fascistisation and management of theatre in Italy, 1925-1943. In G. Berghaus (Ed.), Fascism and Theatre: Comparative Studies on the Aesthetics and Politics of Performance in Europe, 1925-1945 (pp. 96-112). Providence, Oxford: Berghahn Books.

Tinterri, A. (2009). Arlecchino a Palazzo Venezia. Momenti di teatro nell'Italia degli anni Trenta. Perugia: Morlacchi.

Zancan, M., \& Pipitone, C. (2006). L'Archivio Sibilla Aleramo. Guida alla consultazione. Roma: Fondazione Istituto Gramsci onlus.

Zawiszewska, A. (Eds.). (2015). Granice Nałkowskiej. Szczecin \& Warszawa: Wydawnictwo Naukowe Uniwersytetu Szczecińskiego \& Wydawnictwo Feminoteki.

Riassunto: Sibilla Aleramo (1876-1960) ed Emilia Szenwicowa (1889-1972), giornalista e traduttrice polacca, si conobbero nel 1927: la scrittrice italiana sperava che Szenwicowa avrebbe tradotto in italiano il suo romanzo Amo dunque sono. Un anno dopo, Aleramo trascorse qualche settimana di vacanza a Positano, nella villa della famiglia Szenwic, dove ritornò spesso fino allo scoppio della seconda guerra mondiale. Nel 1931, grazie alla mediazione di Emilia, Aleramo intraprese la traduzione di Dom kobiet, una pièce teatrale di Zofia Nałkowska, basandosi su traduzioni mediatorie in italiano e francese.

Nell'archivio familiare degli eredi della giornalista polacca è stata recentemente ritrovata una collezione di missive aleramiane. Le lettere, per la prima volta pubblicate in frammenti nel presente 
saggio, testimoniano la profonda amicizia che univa le due intellettuali e documentano, tra l'altro, diversi progetti di collaborazione inerenti la traduzione di testi teatrali dal polacco in italiano. I momenti della corrispondenza tra Aleramo e Szenwicowa che si riferiscono al teatro vengono sottoposti nel presente intervento ad un'analisi approfondita.

Parole chiave: Sibilla Aleramo, Zofia Nałkowska, Emilia Szenwicowa, traduzione teatrale, scritture femminili 